\title{
The Reversed Halo Sign: \\ Not Such a Specific Sign of Organising Pneumonia
}

\author{
Inês Oliveira, Joana Carvalho, Rita Rosa, Catarina Barata \\ Pulmonology Department, Hospital Egas Moniz, Centro Hospitalar Lisboa Ocidental, Lisbon, Portugal
}

Received: 10/10/2019

Accepted: 15/10/2019

Published: 18/11/2019

How to cite this article: Oliveira I, Carvalho J, Rosa R, Barata C. The reversed halo sign: not such a specific sign of organising pneumonia. EJCRIM 2019;6: doi:10.12890/2019_001326.

Conflicts of Interests: The Authors declare that there are no competing interest

This article is licensed under a Commons Attribution Non-Commercial 4.0 License

\section{ABSTRACT}

The reversed halo sign is defined as a focal rounded area of ground-glass opacity surrounded by a more or less complete ring of consolidation. It is a relatively rare sign and initially considered a specific sign of organising pneumonia. We report the case of a 55-year-old female who was being followed-up in a pulmonology consultation due to a $6 \mathrm{~mm}$ nodule which required vigilance. On a re-evaluation chest CT scan, besides a stable $6 \mathrm{~mm}$ nodule, a $36 \mathrm{~mm}$ mass with the reversed halo sign was diagnosed. The presence of the reversed halo sign misled the multidisciplinary team into the diagnosis of organising pneumonia and initiation of corticotherapy was suggested. However, after further investigation, a final diagnosis of pulmonary tuberculosis was made. Even though this sign is relatively rare, and still considered an important clue to the diagnosis of organising pneumonia in immunocompetent patients, other causes must be excluded before starting treatment.

\section{LEARNING POINTS}

- The reversed halo sign (RHS) is defined as a focal rounded area of ground-glass opacity surrounded by a more or less complete ring of consolidation. It is a relatively rare sign, and still considered an important clue to the diagnosis of organising pneumonia (OP). However, the RHS has been described in other pulmonary diseases.

- The diagnosis of OP depends upon the demonstration of typical histopathologic features, usually through lung biopsy, and exclusion of other diseases which led, in our case, to a final diagnosis of pulmonary tuberculosis.

\section{KEYWORDS}

Reversed halo sign, organising pneumonia, pulmonary tuberculosis

\section{INTRODUCTION}

The reversed halo sign (RHS) was initially considered to be a specific sign of organising pneumonia (OP). However, it has been described in a variety of pulmonary diseases. We report a case of an unexpected diagnosis of pulmonary tuberculosis (PT) that was initially thought to be OP due to the presence of this sign, reinforcing the importance of excluding other possible aetiologies when the RHS is present.

\section{CASE PRESENTATION}

A 55-year-old Caucasian female with an active smoking history of 25 pack-years was referred to our pulmonology department due to complaints of chronic intermittent rhinitis and for vigilance of a $6 \mathrm{~mm}$ nodule on the chest CT scan in the right upper lobe. Imaging surveillance was maintained but 12 months after the initial chest CT scan, in addition to a stable $6 \mathrm{~mm}$ nodule on the right upper lobe, a heterogeneous and irregular $36 \mathrm{~mm}$ mass, with a central hypodense zone (RHS), was diagnosed on the apico-posterior region of the left upper lobe (Fig. 1) 

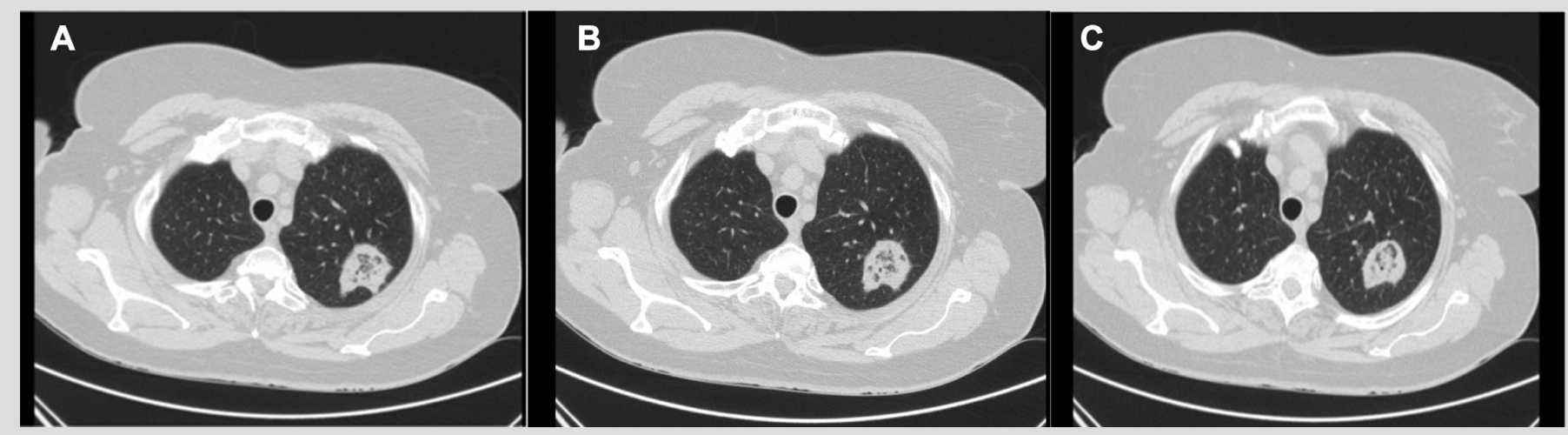

Figure 1. Chest CT scan showing a $36 \mathrm{~mm}$ mass with a central hypodense zone, surrounded by a ring of consolidation (reversed halo sign) on the apico-posterior region of the left upper lobe

The patient had no respiratory or constitutional symptoms and denied complaints suggestive of connective tissue disease. Her husband had been treated, 6 months earlier, for PT but her contact screening was negative. She had no prior relevant medical history and was being treated for her rhinitis with budesonide nasal spray $100 \mathrm{mcg}$ and levocetirizine $5 \mathrm{mg}$.

The general examination was unremarkable, except for a pale nasal mucosa.

Laboratory analysis showed an increase in haemoglobin levels, $16.2 \mathrm{~g} / \mathrm{dl}$ (normal 12-15 g/dl), probably due to the patient's smoking history, with no other relevant changes, particularly regarding inflammatory markers, which were normal. Due to her smoking history, a panel of tumour markers was requested (CEA, CA 19.9, CA 15.3, CA 125, CYFRA-21, NSE and SCC) which were also normal. Serologic tests for hepatitis B, C and HIV were negative and autoimmune antibodies (anti-CCP, ANAs and ANCAs) were within normal values. Pulmonary function tests revealed small airway obstruction with a normal diffusing capacity for carbon monoxide.

The case was discussed in a multidisciplinary team meeting. Given the lack of symptoms, the appearance of a relatively large mass over a short period of time (which made the diagnosis of cancer less likely) and the presence of the RHS on the chest CT scan, OP was considered the most likely diagnosis and initiation of corticotherapy was suggested.

Before starting corticotherapy, in order to rule out alternative causes and confirm the diagnosis, a bronchofibroscopy with bronchoalveolar lavage (BAL) and a percutaneous CT-guided transthoracic needle biopsy of the lung were performed. Pathological analysis of the lung biopsy revealed necrotic tissue. The BAL findings were nonspecific: a total of $63 \mathrm{ml}$ (out of $150 \mathrm{ml}$ ) of opaque fluid was recovered with a total cell count of 494 cells/mcl with a macrophage predominance (94\%). Microbiological and cytological analyses of the BAL fluid were negative for infectious or neoplastic disease. However, bronchial secretions collected during the bronchofibroscopy showed Mycobacterium tuberculosis (MT), susceptible to all first-line drugs (isoniazid, rifampin, pyrazinamide and ethambutol).

The patient had the final diagnosis of PT and not OP, as initially suspected.

She was referred to a specialised tuberculosis center and the recommended treatment for drug-susceptible PT was initiated. The patient completed a 2-month intensive phase with 4 drugs (isoniazid $300 \mathrm{mg} /$ day, rifampin $600 \mathrm{mg} /$ day, pyrazinamide 1,500 mg/day and ethambutol $1,200 \mathrm{mg} /$ day) and is now completing the 4-month continuation phase with: isoniazid $300 \mathrm{mg} / \mathrm{day}$ and rifampin $600 \mathrm{mg} / \mathrm{day}$. She remains clinically stable, asymptomatic and without adverse effects arising from the medication. A re-evaluation chest CT scan was performed and showed imagiological improvement, confirming the diagnosis of PT.

\section{DISCUSSION}

The RHS is defined as a focal rounded area of ground-glass opacity surrounded by a more or less complete ring of consolidation ${ }^{[1]}$. It is a relatively rare sign and it was first described in 1996, in 2 cases of cryptogenic OP. It was initially considered to be a specific sign of OP. However, it was subsequently described in a variety of pulmonary diseases ${ }^{[1,2]}$, both infectious and non-infectious, including tuberculosis. Some authors are attempting to better characterise this sign, in order to aid differential diagnosis, and they have found that the presence of nodules on the wall of or within the halo (nodular RHS) is generally seen in active granulomatous disease, such as tuberculosis ${ }^{[3]}$. Nevertheless, the presence of the RHS is still considered an important clue to the diagnosis of OP, especially in immunocompetent patients ${ }^{[4,5]}$. This was the reason why, in the presented case, this finding in the chest CT scan misled the radiologists and pulmonologists into thinking that OP was the final diagnosis. 
OP is a type of diffuse interstitial lung disease that affects the distal bronchioles, respiratory bronchioles, alveolar ducts and alveolar walls. The diagnosis of OP relies on the demonstration of typical histopathologic features, usually through lung biopsy, and exclusion of other diseases ${ }^{[6]}$. This was the reason why a bronchofibroscopy with BAL and a percutaneous CT-guided transthoracic needle biopsy of the lung were performed in our patient, which gave us the final, and unexpected, diagnosis of PT.

PT is an infectious disease caused by MT. Tuberculosis may affect any organ but lungs are the major site of infection ${ }^{[7]}$. The diagnosis of PT is definitively established by isolation of MT from a bodily secretion or tissue ${ }^{[8]}$. According to international recommendations, the preferred regimen for treating adults with drug-susceptible PT is a regimen consisting of an intensive phase of 2 months of isoniazid, rifampin, pyrazinamide and ethambutol, followed by a continuation phase of 4 months of isoniazid and rifampin as was established in the presented case $^{[9]}$.

Our patient had a history of previous contact with her husband who had been diagnosed recently with PT. However, she had undergone a contact screening which was negative, making the diagnosis of PT, from our point of view, less likely. This, and the presence of the RHS on the chest CT scan, misled the final diagnosis.

Chest CT scans are a very helpful tool in current respiratory medicine, playing a key role in establishing the diagnosis. However, in our case, the imagiologic features have incorrectly led to thinking of an OP diagnosis. This case reflects the importance of histopathologic confirmation of suspected OP, when the RHS is present, which in our case, led to the diagnosis of PT.

\section{REFERENCES}

1. Zhan X, Zhang L, Wang Z, Jin M, Liu M, Tong Z. Reversed halo sign: presents in different pulmonary diseases. PLOS ONE 2015;10:e0128153.

2. Godoy MC, Viswanathan C, Marchiori E, Truong MT, Benveniste MF, Rossi S, et al. The reversed halo sign: update and differential diagnosis. Br J Radiol 2012;85:1226-1235.

3. Marchiori E, Zanetti G, Hochhegger B. Reversed halo sign. J Bras Pneumol 2015;41:564.

4. Faria IM, Zanetti G, Barreto MM, Rodrigues RS, Araujo-Neto CA, Pereira e Silva JL, et al. Organizing pneumonia: chest HRCT findings. J Bras Pneumol 2015;41:231-237.

5. Marchiori E, Zanetti G, Escuissato DL, Souza A, Meirelles G, Fagundes J, et al. Reversed halo sign high-resolution CT scan findings in 79 patients. Chest 2012;141:1260-1266.

6. King TE. Cryptogenic organizing pneumonia. UpToDate 2017. Available online: https://www.uptodate.com/contents/cryptogenic-organizing-pneumonia (accessed 23 April 2019).

7. PozniakA. Clinical manifestations and complications of pulmonary tuberculosis. UpToDate 2019. Available online: https://www.uptodate.com/contents/clinical-manifestationsand-complications-of-pulmonary-tuberculosis(accessed 10 June 2019).

8. Bernardo J. Diagnosis of pulmonary tuberculosis in adults. UpToDate 2019. Available online: https://www.uptodate.com/contents/diagnosis-of-pulmonary-tuberculosis-inadults (accessed 10 June 2019).

9. Nahid P, Dorman SE, Alipanah N, Barry PM, Brozek JL, Cattamanchi A, et al. ATS/CDC/IDSA Clinical Practice Guidelines: treatment of drug-susceptible tuberculosis. Clin Infect Dis 2016;63:853-867. 